\title{
Elastografia ARFI uterina em pós-parto de ovelhas Santa Inês: resultados preliminares
}

Renata Sitta Gomes Mariano ${ }^{[]^{*}}$, Daniele dos Santos Rolemberg ${ }^{[a]}$, Amanda Ilkiu ${ }^{[a]}$, Marjury Cristina Maronezi[a], Ana Paula Rodrigues Simões ${ }^{[a]}$, Michelle Lopes Avante ${ }^{[a]}$, Ricardo Andres Ramirez Uscategui[a], Pedro Paulo Maia Teixeira ${ }^{[b]}$, Marcus Antonio Rossi Feliciano ${ }^{[a, c]}$, Wilter Ricardo Russiano Vicente ${ }^{[a]}$

\footnotetext{
[a] Universidade Estadual Paulista (UNESP), São Paulo, SP, Brasil

${ }^{[b]}$ Universidade Federal do Pará (UFPA), Castanhal, PA, Brasil

${ }^{[c]}$ Universidade Federal do Recôncavo da Bahia (UFRB), Cruz das Almas, BA, Brasil
}

*Autor correspondente

e-mail: renata_sgm@hotmail.com

\section{Resumo}

O objetivo deste estudo foi avaliar a rigidez do tecido uterino durante o pós-parto fisiológico em ovelhas Santa Inês, por meio da elastografia-ARFI (Acoustic Radiation Force Impulse). Determinaramse a velocidade da onda de cisalhamento uterina ( $\mathrm{SWV} \mathrm{m} / \mathrm{s}$ ) e a profundidade $(\mathrm{cm})$ da avaliação para três regiões de interesse diferentes da parede uterina em cinco ovelhas hígidas, com gestação e parto fisiológicos. O exame foi feito pela ultrassonografia transabdominal, utilizando o aparelho ACUSON S2000/SIEMENS, transdutor multifrequencial, convexo de 5,0 a 8,0 $\mathrm{MHz}$ e software para análise elastrográfica-ARFI (Siemens, Munich, Alemanha), no pós-parto imediato e a cada 48 horas, durante 30 dias. A análise estatística foi realizada com ajuda do software R (R Project, Viena, Áustria). Inicialmente, comprovaram-se a normalidade e homocedasticidade das variâncias. Posteriormente, compararam-se as observações das regiões avaliadas pelo teste de ANOVA, e correlacionaram-se (teste de Pearson) a SWV e a profundidade desta avaliação com os dias pós-parto. A SWV e a profundidade desta avaliação foram similares entre as observações realizadas ( $P=0,624$ e 0.850 , respetivamente); desta forma, foram utilizadas as médias das três avaliações para a análise da rigidez tecidual com relação ao tempo. Evidenciou-se uma fraca correlação negativa $(r=-0,420 ; P=0.001)$ entre a SWV e os dias pós-parto, assim como entre a profundidade do tecido avaliado e a SWV ( $\mathrm{r}=-0,431 ; \mathrm{P}<0.001)$. Em conclusão, a avaliação elastográfica-ARFI da parede uterina durante o pós-parto fisiológico em ovelhas mostrou-se uma técnica aplicável e com resultados promissores para a avalição não invasiva deste período fisiológico, no qual a rigidez tecidual diminui gradativamente com o passar dos dias. 